'Sección de Reumatología. Departamento de Medicina Oriente. Escuela de Medicina. Universidad de Chile. Santiago, Chile.

${ }^{2}$ Sección de Reumatología. Servicio de Medicina. Hospital del Salvador. Santiago, Chile. ${ }^{3}$ Servicio de Imagenología. Hospital del Salvador. Santiago, Chile.

${ }^{4}$ Departamento de Salud Ocupacional, Corporación Nacional del Cobre. Santiago, Chile.

Trabajo no recibió financiamiento. Los autores declaran no tener conflictos de interés.

Recibido el 11 de julio de 2019, aceptado el 17 de marzo de 2020.

Correspondencia a: Daniel Erlij Opazo Avenida Salvador 364. Santiago, Chile. danerlij@gmail.com

\section{Poliadenopatías de origen reumatológico y las claves del diagnóstico diferencial: Análisis de 19 casos}

\author{
DANIEL ERLIJ ${ }^{1}$, MARÍA CAROLINA CUELLAR ${ }^{2}$, \\ NATALIA BADILLA ${ }^{2}$, PERLA BARROS ${ }^{3}$, IGNACIO MÉNDEZ ${ }^{4}$, \\ ANGELA RIVERA ${ }^{2}$, CAROLINA FOSTER ${ }^{2}$, \\ VERÓNICA WOLFF ${ }^{1,2}$, SUSANA MICHALLAND ${ }^{2}$
}

\section{Lymphadenopathies in patients with rheumatic diseases. Review of 19 cases}

Background: The presence of multiple lymphadenopathies can be a diagnostic challenge. Aim: To describe the clinical, laboratory and imaging characteristics of 19 patients with lymphadenopathies of rheumatologic origin. Material and Methods: Review of medical records of 19 patients aged 16 to 72 years (68\%) with lymphadenopathies presumably secondary to a rheumatic disease. Results: Six patients had systemic lupus erythematosus, six had Sjogren's disease, three had sarcoidosis, two had rheumatoid arthritis, one had IgG4 related disease and one had mixed connective tissue disease. A lymph node biopsy was performed in 11 patients and in eight a lymphoid follicular hyperplasia was found. Systemic symptoms were reported by $68 \%$ of patients. Blood lactate dehydrogenase was elevated only in cases associated with hemolytic anemia. There was no specific or predictable localization of the lymphadenopathies in imaging studies, except in the cases of sarcoidosis. The average size of the lymphadenopathies was 13.5 $m m$ in diameter in short axis and there was no presence of necrosis, calcification, or conglomerate formation. Only one case presented splenomegaly. All patients responded favorably to corticosteroids. Conclusions: Lymphadenopathies associated with rheumatologic diseases can occur in a wide variety of diseases, especially systemic lupus erythematosus and Sjögren's disease. The absence of LDH elevation and splenomegaly and the absence of imaging findings such as conglomerates can orient to a rheumatologic origin.

(Rev Med Chile 2020; 148: 320-326)

Key words: Lupus Erythematosus, Systemic; Lymphadenopathy; Rheumatic Diseases; Sarcoidosis; Sjogren's Syndrome.
E n nuestro organismo existen alrededor de 600 nódulos linfáticos. Generalmente, se denomina adenopatía a un ganglio de consistencia alterada o de tamaño mayor a $1 \mathrm{~cm}$, lo cual puede variar según la región anatómica ${ }^{1}$. Los tres grupos etiológicos más relevantes de poliadenopatías corresponden a infecciones, neoplasias y enfermedades reumatológicas. Si bien la ecografía es una excelente aproximación al estudio de las adenopatías, la tomografía axial computada (TAC) es el examen que permitirá evaluar la extensión del compromiso ganglionar, en especial aquellas adenopatías que no son pesquisables al examen físico, como las mediastínicas y las intraabdominales². 
Si bien la patología oncológica abarca menos de $1 \%$ de las causas de adenopatías ${ }^{1}$, sigue siendo el principal "fantasma" para el médico clínico, quien tiende a centrar su estudio en dicho ámbito. Cuando las adenopatías son localizadas, dicha opción es menos dificultosa de estudiar, pero cuando se trata de un caso de poliadenopatías generalizadas, surge con enorme fuerza la opción de los linfomas, patología que solo da respiro al clínico cuando la biopsia la descarta. Existen estudios que describen como predictores de etiología neoplásica la presencia de lactato deshidrogenasa (LDH) aumentada, esplenomegalia y a la localización tanto supraclavicular como intraabdominal ${ }^{3}$.

Del punto de vista infeccioso, el virus Epstein Barr, la tuberculosis ganglionar (diagnóstico diferencial por excelencia de los linfomas) y la enfermedad por virus de la inmunodeficiencia humana (VIH) lideran las etiologías de poliadenopatías generalizadas. Este último con la dificultad agregada de la mayor asociación a linfomas.

La presencia de síntomas B, es decir, baja de peso, fiebre y sudoración nocturna, tan característica de los linfomas, es posible de ver tanto en patologías infecciosas como tuberculosis, como en enfermedades reumatológicas, por lo que su presencia no debe ser un distractor ${ }^{4}$.

Si la etiología es reumatológica, la biopsia ganglionar muestra hallazgos histológicos que pueden incluir hiperplasia folicular, paracortical o sinusal, inflamación granulomatosa o linfadenitis aguda ${ }^{1}$. La hiperplasia nodular linfoide, interpretada como adenopatía reactiva, genera un escenario que desplaza el velo del linfoma de los ojos del médico y abre un escenario en el cual las causas reumatológicas adquieren mayor relevancia, destacando dentro de las posibilidades, el lupus eritematoso sistémico (LES), el síndrome de Sjögren (SS), la enfermedad de Still, la enfermedad relacionada a IgG4 (ER-IgG4), la enfermedad mixta del tejido conectivo (EMTC), la sarcoidosis y la artritis reumatoídea, entre otras.

En relación al LES, la presencia de linfadenopatías está descrita hasta en $34 \%$ de los casos, con series que llegan a una cifra de $50 \%$, muchas veces relacionando su presencia a la actividad de la enfermedad, con mayor asociación a síntomas constitucionales, mucocutáneos, presencia de esplenomegalia, anticuerpos anti-DNA e hipocomplementemia ${ }^{5}$. La ER-IgG4 puede presentar adenopatías localizadas o generalizadas. Un detalle relevante es la usual normalidad o la discreta elevación de los niveles de LDH en sangre 6 .

En el SS, la presencia de poliadenopatías puede llegar a $8 \%$ de los casos, con la especial relevancia que el linfoma no solo es diagnóstico diferencial, sino también un riesgo a largo plazo ${ }^{7}$.

Este trabajo pretende caracterizar del punto de vista clínico, de laboratorio e imagenológico, estos cuadros de poliadenopatías que antiguamente eran conocidos como pseudolinfomas, todos ellos con patologías reumatológicas como causal, en 19 pacientes del Hospital del Salvador, con el objetivo de contrastar los hallazgos con las escasas descripciones presentes en la literatura y a su vez, evaluar la existencia de aspectos que permitan evaluar un mayor o menor riesgo de linfoma en un paciente con poliadenopatías.

\section{Material y Método}

\section{Pacientes}

Este trabajo fue aprobado por el Comité de Ética local. Se realizó un análisis retrospectivo de pacientes adultos evaluados en el Hospital del Salvador entre los años 2010 y 2019, todos ellos con poliadenopatías en diversos escenarios clínicos, las cuales resultaron estar en un contexto reumatológico. Los diagnósticos finales se llevaron a cabo a través de los criterios de clasificación actualmente vigentes para cada una de las patologías.

Se revisaron las fichas clínicas de dichos pacientes, recopilando los siguientes datos al momento del diagnóstico: demográficos (edad y género), clínicos (presencia de síntomas B, es decir, fiebre, sudoración nocturna y baja de peso significativa, la presencia de afección cutánea, artritis y serositis para los casos de LES, manifestaciones sistémicas en los casos de SS), laboratorio general y específico (velocidad de eritrosedimentación [VHS], niveles de lactato deshidrogenasa [LDH], hemograma y serología reumatológica), todos con serología viral (VIH, hepatitis B y C) negativos. Se evaluaron también aspectos imagenológicos mediante tomografía axial computada analizados por una misma radióloga (localización, morfología, presencia de necrosis, realce, calcificaciones, diámetro mayor en eje corto y hallazgos extraganglionales) y hallazgos histológicos en las biopsias quirúrgicas de ganglios.

Se realizó un análisis general con la presencia de síntomas B, VHS y LDH, una evaluación ima- 
genológica general y un análisis particular de los pacientes con LES, por un lado, y aquellos con SS, por otro.

\section{Resultados}

\section{Características clínicas}

Se recopilaron 19 pacientes (Tabla 1), 13 de ellos mujeres $(68 \%)$. La edad promedio fue 46 años (fluctuando entre 16 y 72 años). Los diagnósticos fueron ER-IgG4 en un caso, EMTC en un caso, artritis reumatoídea seropositiva (AR) en dos casos, sarcoidosis en tres casos, LES en seis casos y SS en seis casos. De los pacientes analizados, seis (32\%) llegaron en primera instancia a hematología por sospecha de linfoma, el cual fue descartado a través de biopsia ganglionar. En 11 pacientes fue posible la realización de biopsia, evidenciando una hiperplasia folicular linfoide de aspecto reactivo en 8 de ellos y en los restantes, los hallazgos característicos de la sarcoidosis y de la ER-IgG4.

En el aspecto clínico, 13 pacientes (68\%) presentaron síntomas $\mathrm{B}$, ahora, solo uno de ellos (portadora de un SS) presentó las tres manifestaciones respectivas al mismo tiempo. La VHS mostró una gran dispersión, la cual se mantiene luego del análisis específico por patología, con valores entre 4 y $140 \mathrm{~mm} / \mathrm{h}$ y un promedio de $50 \mathrm{~mm} / \mathrm{h}$. Respecto a los niveles de LDH, solo 2 pacientes superaron el límite superior.

En la evaluación imagenológica (Tabla 2), se observó que 15 pacientes (79\%) presentaban más de un sitio de compromiso adenopático, siendo

Tabla 1. Descripción general de casos

\begin{tabular}{|ccccccc|}
\hline $\mathbf{n}$ caso & Sexo & $\begin{array}{c}\text { Edad } \\
\text { (años) }\end{array}$ & $\begin{array}{c}\text { Diagnóstico } \\
\text { principal }\end{array}$ & $\begin{array}{c}\text { Presencia de } \\
\text { síntomas B }\end{array}$ & $\begin{array}{c}\text { VHS } \\
(\mathbf{m m} / \mathbf{h})\end{array}$ & $\begin{array}{c}\text { LDH (Normal: } \\
\mathbf{2 3 0 - 4 6 0} \text { UI/L) }\end{array}$ \\
\hline 1 & M & 35 & AR & Sí (BP) & 60 & 185 \\
\hline 2 & M & 57 & AR & No & 24 & $(-)$ \\
\hline 3 & $F$ & 51 & EMTC & No & 6 & 141 \\
\hline 4 & M & 40 & ER-IgG4 & No & 46 & 131 \\
\hline 5 & M & 54 & Sarcoidosis & Sí (SN) & 58 & 180 \\
\hline 6 & $F$ & 37 & Sarcoidosis & Sí (F, BP) & 27 & 170 \\
\hline 7 & M & 60 & Sarcoidosis & No & 55 & 230 \\
\hline 8 & $F$ & 28 & LES & Sí (BP) & 22 & 197 \\
\hline 9 & $F$ & 55 & LES & Sí (BP) & 140 & 509 \\
\hline 10 & $F$ & 16 & LES & Sí (SN) & 27 & 389 \\
\hline 11 & $F$ & 41 & LES & Sí (F) & 101 & 240 \\
\hline 12 & $F$ & 28 & LES & Sí (F) & 68 & 195 \\
\hline 13 & $F$ & 30 & LES & Sí (F) & 47 & 632 \\
\hline 14 & $F$ & 68 & Sjögren & Sí (BP, SN) & 50 & 311 \\
\hline 15 & $F$ & 43 & Sjögren & Sí (BP) & 104 & 157 \\
\hline 16 & $F$ & 49 & Sjögren & Sí (BP) & 91 & 265 \\
\hline 17 & $F$ & 72 & Sjögren & No & 4 & 215 \\
\hline 18 & $F$ & 49 & Sjögren & Sí (BP, F, SN) & 15 & 134 \\
\hline 19 & F & 55 & Sjögren & No & 9 & 205 \\
\hline
\end{tabular}

F: femenino. M: masculino. AR: artritis reumatoide. EMTC: enfermedad mixta del tejido conectivo. ER-IgG4: enfermedad relacionada con IgG-4. VHS: velocidad de eritrosedimentación. LDH: lactato deshidrogenasa, LES: lupus eritematoso sistémico. F: fiebre, BP: baja de peso, SN: sudoración nocturna. 


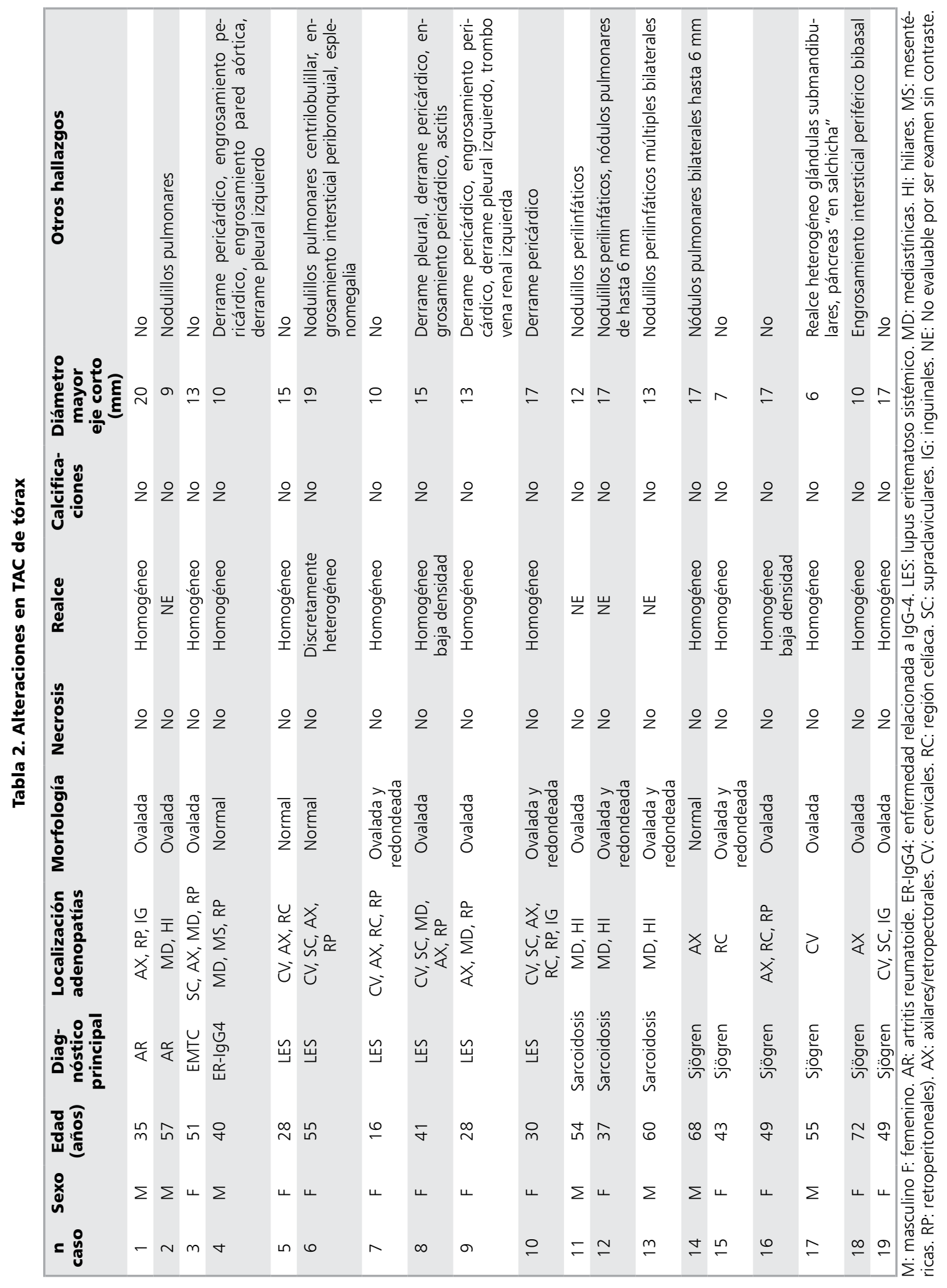


el axilar el más frecuente, con 11 casos (58\%), seguido por el retroperitoneal, con 9 pacientes $(47 \%)$. La morfología predominante fue la ovalada en 15 casos (79\%), mientras que ninguno mostró necrosis ni calcificaciones. Catorce casos (74\%) presentaron realce homogéneo al medio de contraste. Sin embargo, este aspecto no pudo ser evaluado en 4 pacientes, por la ausencia de contraste en el examen. El diámetro mayor en eje corto fue en promedio de $13,5 \mathrm{~mm}$, siendo el menor valor $6 \mathrm{~mm}$ y el mayor $20 \mathrm{~mm}$. Los hallazgos asociados, en general, se relacionaron a su enfermedad de base. No hubo presencia de conglomerados adenopáticos en ninguno de los casos. Solo en un caso se encontró esplenomegalia.

El análisis de los 6 pacientes con LES (Tabla 3) mostró la presencia frecuente de anemia de diversas etiologías en 5 de ellos, la positividad al anticuerpo Ro, Sm y RNP en 4 casos y el hallazgo de complemento $\mathrm{C} 4$ disminuido en 5 pacientes.

Respecto a los 6 pacientes con SS (Tabla 4), solo la mitad presentó la serología clásica con ANA $(+)$, ENA $(+)$ para Ro y La y factor reumatoídeo $(+)$. En aquellos que no la presentaba, la biopsia de glándulas salivales menores apoyó el diagnóstico.

En relación al tratamiento, todos los pacientes recibieron corticoides a dosis variables, que fueron desde $15 \mathrm{mg} /$ día de prednisona hasta pulsos de metilprednisolona intravenosos cuando el contexto clínico lo ameritó, como en el caso con LES que cursó con anemia hemolítica autoinmune. En todos los casos se produjo respuesta favorable al control imagenológico. Nueve pacientes $(47 \%)$ presentaron resolución completa de las adenopatías, 5 (26\%) presentaron una reducción de tamaño mayor a $50 \%$ y en los 5 restantes, no hubo control imagenológico, ya que, al estar en un contexto autoinmune multiorgánico, se utilizaron otras variables de seguimiento. Todos los pacientes siguen actualmente en control.

\section{Discusión}

En nuestro estudio, los pacientes con LES y SS suman el 64\% de los casos, siendo concordante con la descripción en la literatura en relación a ser las dos patologías reumatológicas con mayor asociación a adenopatías. Debe tenerse en consideración que, muchas veces, las adenopatías en estos pacientes son un hallazgo imagenológico $y$, por tanto, no hay duda que la presencia de las mismas podría ser mucho mayor si se realizaran estudios radiológicos de rutina.

$\mathrm{El}$ análisis de los resultados, con la limitante del escaso número de pacientes, permite concluir algunos aspectos relevantes. En primer lugar, la edad de los pacientes es sumamente variable, no solo en el análisis general de los mismos, sino también cuando se analiza cada patología por separado, impidiendo establecer una tendencia. Lo mismo ocurre con los valores de VHS. Ahora, el aspecto más llamativo es la normalidad de los niveles de LDH, con la sola excepción de dos pacientes que explican el alza por la hemólisis concomitante. Esto resulta interesante en cuanto a que, generalmente, en los linfomas suelen elevarse los niveles de la enzima y esto, a su vez, se relaciona a la masa tumoral. Si consideramos el amplio número y distribución de las adenopatías en los pacientes descritos con nula elevación de LDH, el valor predictivo negativo de este marcador para la etiología linfomatosa resulta de gran utilidad. En los pacientes que no se realizó biopsia ganglionar, la claridad diagnóstica basada en aspectos clínicos y serológicos, asociado a la buena respuesta a tratamiento inmunosupresor mantenida en el tiempo, permitió apoyar la etiología inmunológica.

Respecto a la imagenología, no existió un patrón de distribución identificable, con la sola excepción de los 3 pacientes con sarcoidosis, en los cuales se presentó el clásico compromiso mediastínico e hiliar. Vale la pena destacar que la localización supraclavicular, tan temida por su reputación de malignidad, estuvo presente en 5 casos (26\%) de manera transversal a los distintos diagnósticos, lo cual pone en cuestión su clásica interpretación. Lo mismo ocurre con los intraabdominales. Si bien la ausencia en todos los casos de necrosis no resulta de gran utilidad para la diferenciación con linfoma, pues estos pocas veces la presentan, la presencia de la misma debe orientar a etiología infecciosa o neoplásica de tipo metastásica. Las calcificaciones son muy características de la sarcoidosis, a pesar de lo cual, estuvo ausente en los 3 casos con dicha enfermedad. El realce homogéneo de una adenopatía al contraste solo hace menos probable la etiología infecciosa, pero no descarta el linfoma. En cuanto al tamaño de las adenopatías, si bien ningún caso superó los $20 \mathrm{~mm}$ en eje corto, esto no permite hacer la diferencia con procesos linfoproliferativos, sin embargo, la presencia de 

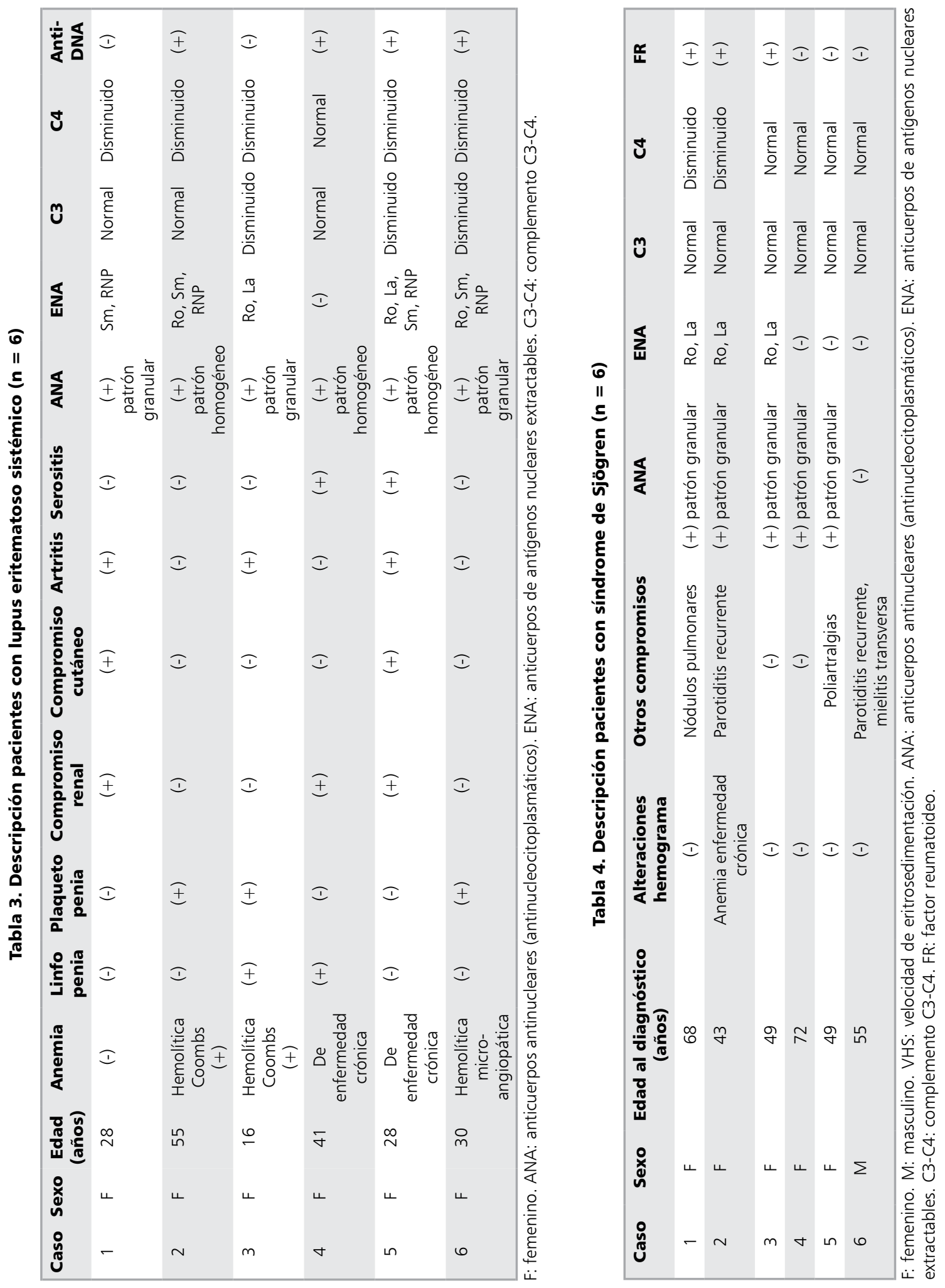
conglomerados ganglionares sí resulta de utilidad, ya que los linfomas tienden a formarlos, no así las patologías reumatológicas, razón por la cual esta característica no se observó en ninguno de los pacientes del estudio. Por último, la existencia de otras manifestaciones sistémicas puede ser de gran valor, ya que hallazgos como un derrame y engrosamiento pericárdico en este escenario, en especial cuando se trata de una mujer entre $20 \mathrm{y}$ 40 años de edad, debe hacer plantear un LES. En este mismo contexto, los nodulillos pulmonares, que si bien por sí solos no constituyen un aporte al diagnóstico diferencial, se transforman en un detalle clave cuando su distribución es perilinfática, sugiriendo fuertemente la sarcoidosis como etiología del cuadro.

El análisis específico de los pacientes con LES deja ver algunos detalles interesantes, como la presencia de anemia en casi todos los casos y la alta frecuencia de anticuerpos antiSm, RNP y Ro. Ahora, el reducido número de pacientes impide obtener mayores conclusiones al respecto, como la valoración de la hipocomplementemia y la presencia de anticuerpos anti-DNA. Lo mismo ocurre con los casos de SS. Cabe destacar que solo un paciente presentó esplenomegalia, siendo una mujer con LES y anemia hemolítica autoinmune, situación que permite explicar dicho hallazgo.

En suma, de los tres predictores de etiología neoplásica en poliadenopatías descritos en la literatura, fue posible comprobar que en los casos de causa reumatológica, la ausencia de esplenomegalia y de elevación de LDH (con las excepciones ya justificadas por la presencia de anemia hemolítica) resultaron una constante, mientras que la localización supraclavicular e intraabdominal no fue de utilidad para diferenciar etiologías.
Este trabajo tiene la fortaleza de ser el único estudio conocido que analiza un grupo de pacientes con distintas enfermedades reumatológicas asociadas a poliadenopatías. Su principal aporte es ser la base de futuras investigaciones que, con un mayor número de casos, permita obtener información estadísticamente significativa que haga más fácil, al clínico y al radiólogo en un trabajo conjunto, el diagnóstico diferencial de las poliadenopatías.

\section{Referencias}

1. Mohseni Sh, Shojaiefard A, Khorgami Z, Alinejad Sh, Ghorbani A, Ghafouri A. Peripheral Lymphadenopathy: Approach and Diagnostic Tools. Iran J Med Sci Supplement March 2014; 39 (2): 158-70.

2. Steinkamp HJ, Cornehl M, Hosten N, Pegios W, Vogl T, Felix R. Cervical lymphadenopathy: ratio of long- to short-axis diameter as a predictor of malignancy. Br J Radiol 1995; 68: 266-70.

3. Akinci S, Silay K, Hacibekiroglu T, Ulas A, Basturk A, Bakanay SM, et al. The predictive value of epidemiological characteristics, clinical and laboratory findings in adult lymphadenopathy etiology. Eur Rev Med Pharmacol Sci 2015; 19: 2973-77.

4. Habermann TM, Steensma DP. Lymphadenopathy. Mayo Clin Proc 2000; 75: 723-32.

5. Melıkoglu MA, Melıkoglu M. The clinical importance of lymphadenopathy in systemic lupus erythematosus. Acta Reumatol Port 2008; 33: 402-6.

6. Palazzo E, Palazzo C, Palazzo M. IgG4-related disease. Joint Bone Spine 2014; 81 (1): 27-31.

7. Malladi AS, Sack KE, Shiboski SC, Shiboski CH, Baer AN, Banushree R, et al. Primary Sjögren's syndrome as a systemic disease: a study of participants enrolled in an international Sjögren's syndrome registry. Arthritis Care Res 2012; 64 (6): 911-8. 